Int. J. Dev. Biol. 49: 309-315 (2005)

doi: $10.1387 / \mathrm{ijdb} .041958 \mathrm{~mm}$

\title{
Synchronised cycling gene oscillations in presomitic mesoderm cells require cell-cell contact
}

\author{
MIGUEL MAROTO\#, J. KIM DALE\#, MARY-LEE DEQUÉANT, ANNE-CÉCILE PETIT and OLIVIER POURQUIÉ* \\ Laboratoire de Genetique et de Physiologie du Developpement, Institut de Biologie du Developpement de Marseille, CNRS-INSERM- \\ Universite de la Mediterranee-AP de Marseille, Campus de Luminy, Marseille, France
}

\begin{abstract}
Segmentation of the vertebrate body axis is initiated early in development with the sequential formation of somites. Somitogenesis is temporally regulated by a molecular oscillator, the segmentation clock, which acts within presomitic mesoderm (PSM) cells to drive periodic expression of the cyclic genes. We have investigated the kinetics of the progression of cycling gene expression along the PSM. Here we show that c-hairy1 and c-hairy2 mRNA expression traverses the PSM in an entirely progressive manner and that both these genes and $c$-Lfng maintain a similar anterior limit of expression during each cycle. However, some differences are seen regarding both the onset of a new oscillation of these genes and the duration of their expression in the caudal PSM. We also investigated whether oscillating cyclic gene expression in the PSM is entirely cell autonomous. We find that while small PSM explants are still able to maintain their oscillation schedule, once they are dissociated, PSM cells are no longer able to maintain synchronous oscillations. The results imply that cell communication or a community effect is essential for the normal pattern of cyclic gene expression in these cells.
\end{abstract}

KEY WORDS: embryo, oscillation, presomitic mesoderm, cyclic gene, synchronisation

\section{Introduction}

The segmented body plan of the vertebrate embryo has been the object of study for many decades and yet the mechanisms that converge to orchestrate this metameric architecture are only now beginning to be understood, reviewed in (Bessho and Kageyama, 2003, Maroto and Pourquié, 2001, Pourquié, 2003). Segmentation of the paraxial mesoderm from the mesenchymal presomitic mesoderm (PSM), which lies either side and juxtaposed to the caudal neural tube, into bilaterally symmetrical epithelial somites, is the earliest manifestation of this metamerism. This process is under strict temporal control which led to a number of models postulating that the species-specific time interval between somitic boundary formation was controlled by an intrinsic oscillator mechanism acting within the PSM cells, reviewed in (Dale and Pourquié, 2000). A series of embryological studies in the chick, mouse and zebrafish have since revealed this is indeed the case at the molecular level. The initial discovery of the oscillating mRNA expression of $c$-hairy1 in the chick PSM was the first demonstration that PSM cells possess oscillatory properties, which have been attributed to a molecular clock functioning within these cells, termed the segmentation clock (Palmeirim et al., 1997). This dynamic mRNA expression profile in the PSM has subsequently been shown to be shared by a variety of genes in chick, mice and zebrafish, nearly all of which are related to the Notch signaling pathway. These genes include the bHLH Notchtarget genes mHes1, mHes7, mHey2, her1, her7, c-hairy1, chairy2, c-hey2, the glycosyltransferase Notch modifier $m L$ fng or $c$-Lfng and the Notch ligand' DeltaC (Aulehla and Johnson, 1999, Bessho et al., 2001, Forsberg et al., 1998, Holley et al., 2000, Jiang et al., 2000, Jouve et al., 2000, Leimeister et al., 2000, McGrew etal., 1998, Oates and Ho, 2002, Palmeirim etal., 1997). During one oscillation, the mRNA expression of these genes traverses the PSM caudorostrally as a wave, with a cycle time that matches the periodicity of somite formation. This dynamic expression profile is tissue autonomous since cycling gene expression continues to oscillate on schedule in isolated PSM explants cultured in vitro (Palmeirim et al., 1997). The expression dynamics are not due to cell displacement but rather rely upon

Abbreviations used in this paper: PSM, presomitic mesoderm.

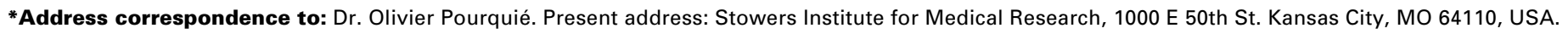
Fax: +1-816-926-2044. e-mail: olp@stowers-institute.org

\#: These two authors contributed equally to this work.
} 
intrinsically coordinated pulses of mRNA expression (Palmeirim et al., 1997). The expression wave does not require the propagation of a caudally derived signal since removal of the caudal domain of a PSM explant does not impede propagation of the wave through the rest of the explant when cultured in vitro (McGrew et al., 1998, Palmeirim et al., 1997). This raises the possibility that cyclical gene expression may be a cell autonomous property of PSM cells.

To date a number of roles have been attributed to the Notch signaling pathway in terms of its function in the somitogenesis process. One role of the segmentation clock could be to generate pulses of Notch activation leading to the periodic and regular array of somitic boundaries. Indeed, the pulsatile expression of the Notch ligand, DeltaC, in fish and the glycosyltransferase, $L f n g$, in mice and chick would clearly generate such a rhythmic activation of the Notch pathway (Aulehla and Johnson, 1999, Forsberg et al., 1998, Jiang et al., 2000, McGrew et al., 1998). A second role invokes Notch as an instrumental component in the synchronization of cycling gene oscillations between neighboring PSM cells, such that the absence of Notch signaling would lead to a gradual desynchronisation of cycling gene expression in this tissue leading to a loss of regular somitogenesis (Jiang et al., 2000). Analyses of fish and mice Notch-signaling mutants as well as Notch loss-of-
A

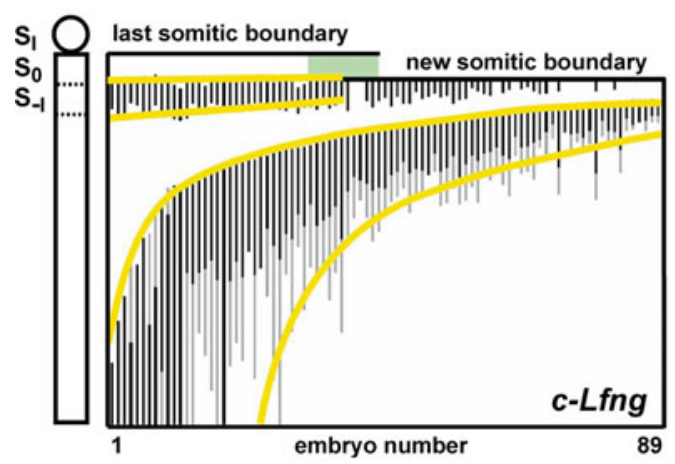

C

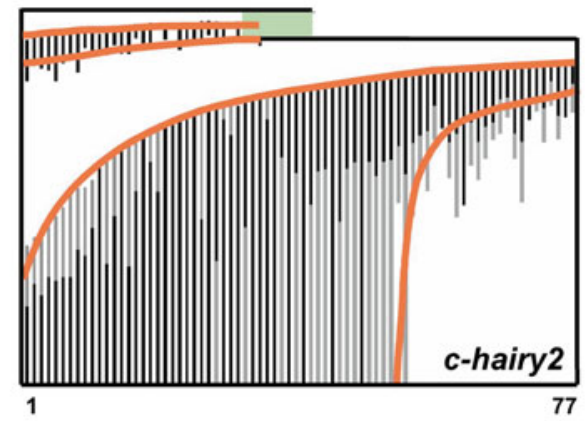

B

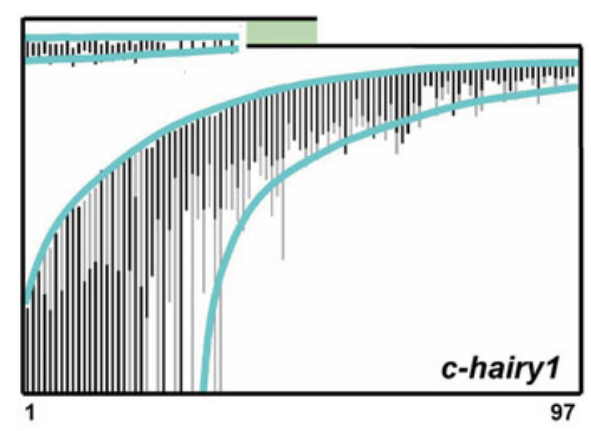

D

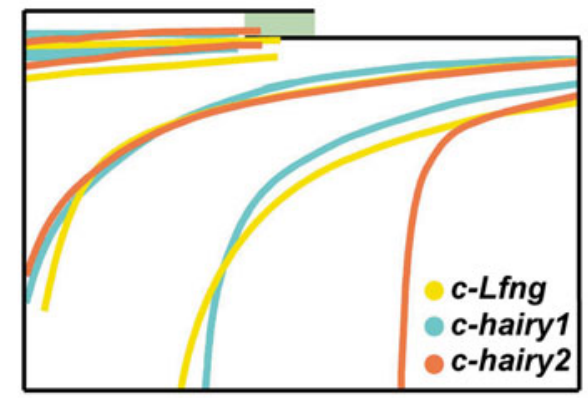

Fig. 1. Comparison of the mRNA expression profiles of c-Lfng, c-hairy1 and c-hairy2 in the chick PSM. (A-C) Analysis of c-Lfng, c-hairy1 and c-hairy2 mRNA expression in 89, 97 and 77 embryos, respectively. Each baralong the x-axis represents one embryo. The $y$-axis represents the extension of gene expression domains along the anteroposterior axis of the PSM, anterior to the top. Darker stripes represent stronger expression domains as compared to lighter bars. The green diagonal striped domain highlights the group of embryos undergoing formation of a new somitic border. The colored lines delimit the anterior and posterior limits for the expression domains of each gene in the PSM. (D) The expression domains for the three genes have been superimposed to compare the kinetics of wave progression in the PSM during one cycle using the window of border formation as a common time-point in the three graphs. function studies in the chick invoke a third more instructive role for this pathway within the clock mechanism. These studies reveal absence of Notch signaling abolishes oscillations of the components of the Notch pathway such as DeltaC and Lfng "thereby placing this pathway potentially within the core mechanism of the oscillator (Bessho et al., 2001, Conlon et al., 1995, Dale et 2002, Hrabe de Angelis et al., 1997, Jiang et al., 2000, Kusumi et al., 1998, Oka et al., 1995, Swiatek et al., 1994, Zhang and Gridley, 1998). These roles are not necessarily mutually exclusive bove levels of the somitogenesis process.

Recent data have revealed that these oscillating expression patterns are, at least in part, likely maintained by negative autoregulatory loops at the level of the Hes/her genes (Bessho et al., encode bHLH transcriptional repressors that can bind their own promotors and thereby shut off their own transcription. Indeed, it was recently shown that periodic repression by oscillating levels $m$ Hes 7 and $m L$ fng (Bessho et al., 2003). In chick, oscillating cLfng protein in the PSM brings about a second level of negative auto-regulation. Misexpression of cLfng in the PSM abolishes expression of all cycling genes including itself (Dale et al., 2003). This phenocopies inhibition of Notch signaling in this tissue, suggesting that oscillating $c$ Lfng protein leads to periodic Notch inhibition. Thus, the main mechanism driving oscillations of these Notchrelated cycling genes appears to involve periodic activation and/or inhibition of the Notch signaling pathway as well as periodic transcriptional repression of the cycling genes themselves.

In this study we carefully investigated the kinetics of the progression of the wave of c-hairy 1 and c-hairy2 mRNA expression along the PSM and compared this to our previous data pertaining to $c$ Lfng expression (Dale et al., 2003). These data suggest that the mRNA expression of all three genes traverses the PSM in an entirely progressive manner and that as the wave moves across the PSM these genes maintain a similar anterior limit of expression. However, some differences are seen with respect to the duration of expression in the caudal PSM and the data also suggest that, at the onset of the cycle in the caudal PSM, c-hairy1 and chairy2 expression slightly precedes that of $c$-Lfing. At the end of the cycle 
as the new somitic border forms, the expression domains of the three genes segregate out to different anteroposterior somitic compartments. We also describe experiments in which we extend some of the initial studies looking at the potential cell autonomy of oscillating cyclic gene expression in the PSM. To that end we dissected the PSM into smaller and smaller fragments and we find that these explants are still able to maintain their oscillation schedule. However, our data suggest that, once dissociated, PSM cells are not able to maintain synchronous oscillations implying that cell communication or a community effect is essential for the normal pattern of cyclic gene expression in these cells.

\section{Results}

The mRNA expression sequence of the clock genes is commonly divided into three phases comprising a broad caudal domain of the PSM (phase I), a narrower domain located in the middle of the PSM (phase II) and a further restricted domain in the rostral most PSM (phase III) (Pourquié and Tam, 2001). It was recently shown that in the case of $c$ - $L$ fng, this sequence traverses the PSM as a continuous progressive wave (Dale et al., 2003). The first aim of this study was to compare the expression kinetics of two other avian cycling genes, $c$-hairy1 and c-hairy2. A large set of stagematched embryos was hybridized with either a $c$-hairy 1 riboprobe or a c-hairy2 riboprobe. The domains of expression and their distance from the last formed somite boundary were measured. The measurements for each individual embryo were plotted in order of the advancing anterior expression limit (Figure1). These data clearly show that the mRNA expression profiles for both $c$ hairy1 and c-hairy2 sweep the PSM caudorostrally in a progressive manner. Moreover, for all three genes, the rate of progression of the wave is clearly much faster in the caudal PSM. These analyses did reveal some differences however between the expression profiles of these three cycling genes. Firstly, the duration of expression in the caudal PSM differs markedly among the three genes. While $c$-hairy 1 and $c$-Lfng mRNAs appear to be degraded fairly rapidly in the caudal PSM, $c$ hairy2 mRNA expression is maintained, albeit at a reduced intensity, in this domain. This caudal domain of c-hairy2 expression is then rapidly degraded towards the end of the cycle. As previously reported, approximately $20-25 \%$ of embryos display a caudal domain of $c$ - $L f$ fng expression, however, there is a slightly higher representation of this phase, (30-35\%), in embryos analyzed for c-hairy1. In the case of c-hairy2, approximately $65-70 \%$ of embryos display the caudal domain of expression. Secondly, the expression domains of the three genes begin to segregate as the wave reaches the rostral PSM.
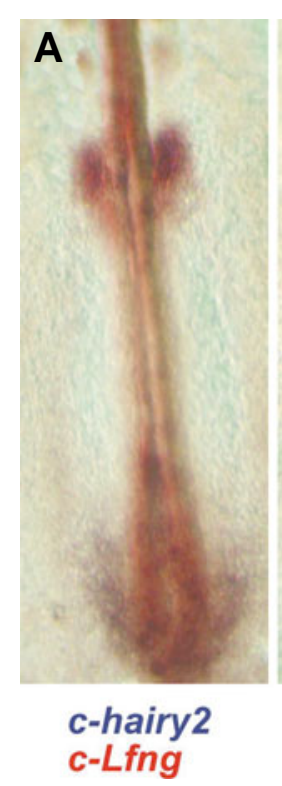
Fast Red.
Thus, once the new border forms $c$-hairy1 and c-hairy2 are located in the posterior compartment of $S_{1}$, the newly formed somite, while $c$-Lfng expression is restricted to the cells of the rostral end of the PSM that now lie in the anterior region of $S_{0}$.

To further compare the expression domains of the three genes, we superimposed the expression domains from the three graphs described above, the point of reference being the window in the cycle during which border formation occurs. The superimposed schema indicates that the advancing position of the anterior front of the expression sequence is shared by all three genes for most of the cycle (Figure 1D). However, this schema suggests that mRNA expression of $c$-hairy 1 and $c$-hairy2 is initiated slightly prior to that of $c$-Lfng in the caudal PSM. This desynchrony would only account for a very short period in the cycle and, once initiated, the anterior front of $c$ - $L$ fng mRNA expression rapidly draws level with that of c-hairy1 and c-hairy2. To confirm this hypothesis we performed double in situ hybridization to directly compare the expression of $c$-Lfng with that of $c$-hairy2 in the same embryo (Figure 2A-E). The majority of embryos showed an overlap in the advancing anterior limit of expression of $c$ - $L$ fng with that of $C$ hairy2 in the PSM (Figure 2C-D). However, in a minority of cases we detected the onset of a caudal expression domain for c-hairy2 while expression of $c$ - $L$ fng was restricted to the narrow stripe in the rostral PSM from the previous cycle (Figure $2 \mathrm{~A}$ ). These results provide additional support for the idea that, while for the most part the mRNA expression profile of these genes traverses the PSM in synchrony, transcriptional activation of the c-hairy 1 and $c$ hairy2 cycling genes appears to be initiated slightly prior to that of $c$-Lfng at the onset of a new oscillation in the caudal PSM.

The second aim of this study was to further evaluate the requirement for cell communication in cyclical gene expression by addressing to what extent these oscillations are an autonomous property of the PSM cells. Initially the PSM from one side of a chick

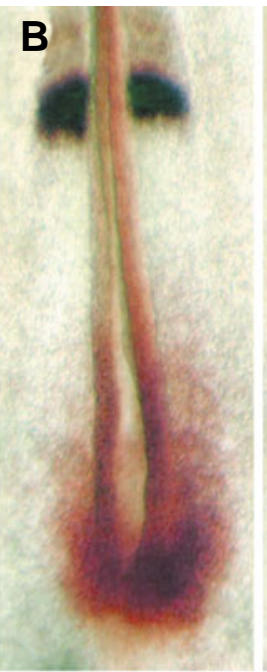
c-hairy2
c-Lfng

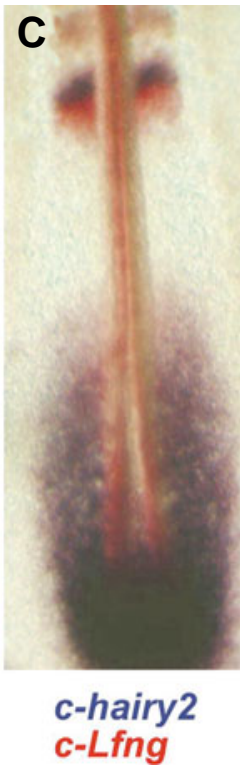

c-hairy2
c-Lfng
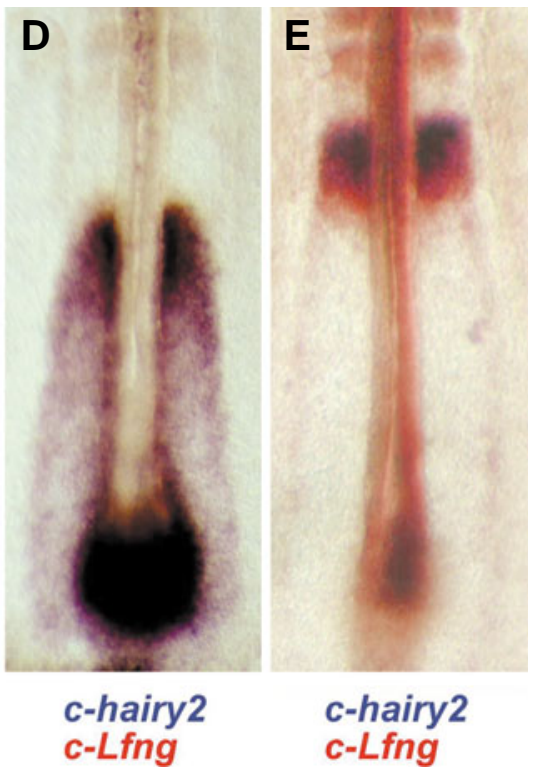

Fig. 2. Double in situ hybridization to compare the expression of $c$-Lfng and c-hairy2 in the chick PSM. $(\mathbf{A}, \mathbf{C}, \mathbf{D}, \mathbf{E})$ c-hairy2 expression revealed in blue with NBT/BCIP and c-Lfng expression revealed in red with Fast Red. (B) c-Lfng expression revealed in blue with NBT/BCIP and c-hairy2 expression revealed in red with 

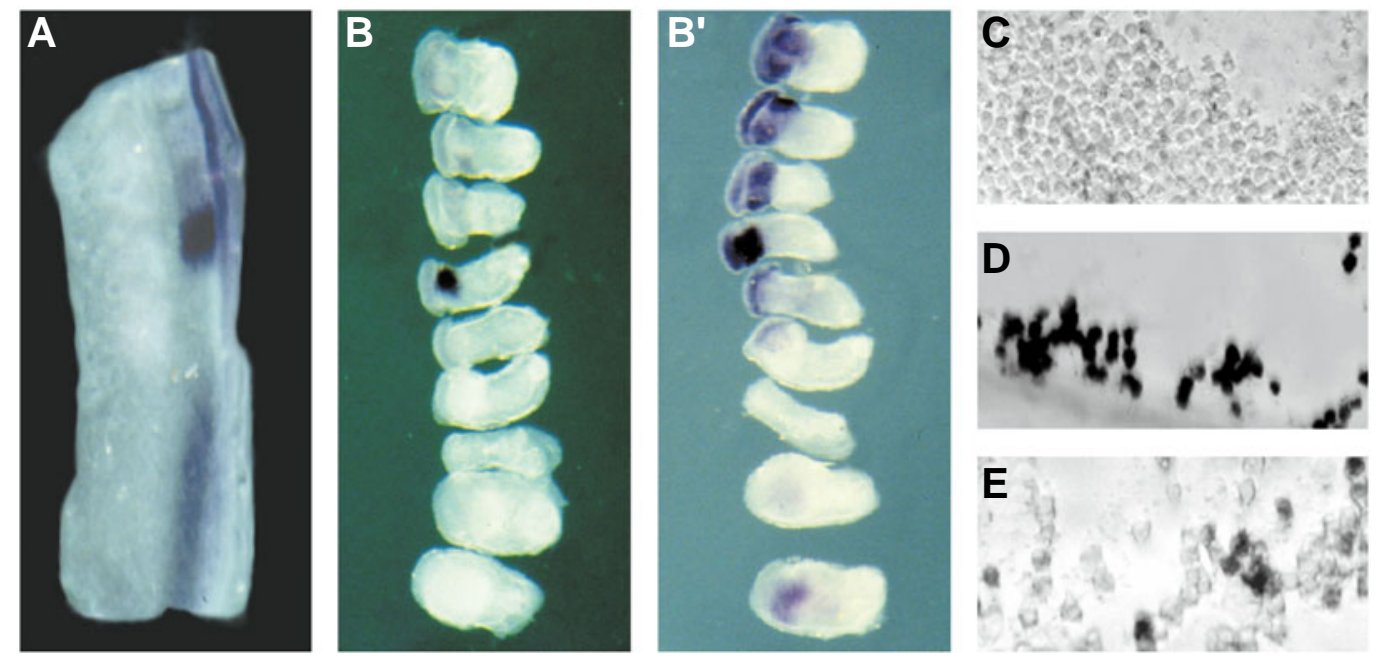

Fig. 3. Cell communication is essential for PSM cells to maintain their correct oscillation schedule. (A-B') c-Lfng expression in PSM explants cultured for 2.5 hours. (A) Intact control half embryo explant.

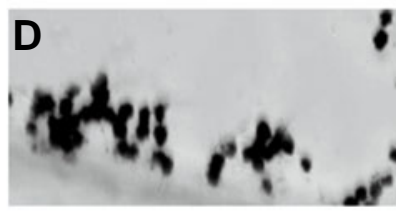
Color revelation was carried out after 2 hours at $37^{\circ} \mathrm{C}$. (B-B') Contralateral half embryo dissected into nine fragments. (B) Expression after $13 \mathrm{~h}$ in revelation buffer at $37^{\circ} \mathrm{C}$. ( $\left.\mathbf{B}^{\prime}\right)$ Expression after 13 h and an overnight

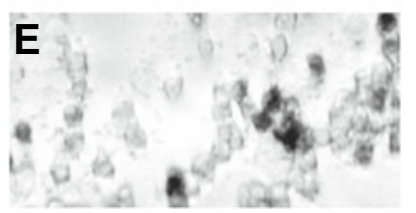
incubation, in revelation buffer, at $37^{\circ} \mathrm{C}$. (C-E) Dissociated PSM cells hybridized in poly-Lysine coated 24well plates for (C) Shh expression, (D) Tbx6L expression or (E) C-Lfng expression.

embryo was dissected into a number of equally sized fragments along the anteroposterior axis, ranging from two to nine fragments. These small explants were then cultured in vitro for the same time as the intact contralateral PSM and were subsequently analyzed by in situ hybridization for expression of $c$-Lfng mRNA. When the PSM was dissected into two, four, six, or even nine fragments and then cultured for two and a half hours in vitro, which corresponds to more than one complete cycle, these small explants were able to continue to oscillate on schedule as compared to the intact control half (Figure3A-B'). However, the smaller the fragments, the more the expression intensity dropped as shown by the extended time required for the color revelation (Figure 3B'). The effect of reduced intensity in the small fragments is most accentuated in the more posterior PSM fragments. When the explants were cultured for a longer time such as six hours, corresponding to four oscillation periods, the explants were still largely able to maintain their normal oscillation schedule as compared to the intact contralateral PSM (data not shown). However, the effect on signal intensity was even more pronounced as judged by color revelation time and, while expression could still be detected in different rostral PSM domains, in some cases a caudal domain of expression could not be detected in the small fragments while the intact control showed expression in this domain. Taken together, these data suggest that these small explants, when cultured in isolation from the rest of the PSM tissue, possess the information necessary to maintain tight temporal control over clock gene expression in the absence of extrinsic signals.

To test the possibility that cyclical gene expression may be a cell autonomous property of PSM cells, PSM tissue was isolated from individual embryos and the cells of the posterior half $(n=5000$ 6000 cells approximately), were dissociated, cultured in vitro for various time periods and then analyzed by in situ hybridization. All cells were negative for Shh (Figure 3C) and positive for the PSM marker Tbx6L (Figure 3D) while the percentage of cells expressing $c$ - $L f n g$ varied in samples coming from different embryos (Figure 3E). Subsequently, dissociated posterior PSM cells from one side of the embryo were split into three pools of approximately 2000 cells each, which were then cultured for different lengths of time. These cell pools were then analyzed by in situ hybridization. If cycling expression of the clock genes were to continue on schedule under this paradigm each pool should be synchronized and in a different phase of the cycle. The intact contralateral PSM was fixed after the first time-point and hybridized for $c$-Lfing expression to serve as a control for which phase of the cycle the embryo was in. The percentage of cells expressing $c$ Lfng in samples coming from the same PSM varied over time, however these values do not appear to correlate with those predicted based on the expression in the intact contralateral control explant (Table1). In some cases the percentage values, in cell pools from one embryo, rose and fell with increased culture time, while in other cases the percentage values fell then rose. Even when comparing samples that were in a similar phase of the cycle at the onset of the experiment, as judged by the expression in the intact control explant half, there was no consistent pattern in the fluctuating percentage values. Thus, in the absence of cellcell communication, the PSM cells continue to express $c$ - $L f$ fing, however, synchrony of the dynamic expression profile among the cells is lost.

\section{Discussion}

Previous studies in the chick, comparing the expression of the clock genes, $c$-Lfng, $c$-hairy1 and $c$-hairy2, two at a time, in two halves of the same embryo reported that they share similar expression kinetics in terms of their activation and anterior progression in the PSM (Freitas et al., 2001, Gajewski et al., 2003, Jouve et al., 2000, McGrew et al., 1998, Oates and Ho, 2002). Here, we have analyzed the expression sequence of these avian genes more precisely. Our results confirm that, for the most part, the three genes share the same anterior limit of expression until they reach the rostral PSM.

There are nevertheless some clear differences between them at several points along the cycle. Strikingly, the transcription of the bHLH genes $c$-hairy1 and c-hairy2 is initiated slightly prior to that of $c$ - Lfng at the onset of a new oscillation in the caudal PSM (Figure 1D). This observation is confirmed by our double in situ hybridisation analysis (Figure 2A). This delay in the initiation of $c$ Lfng transcription might be related with the fact that de novo protein synthesis is required to allow progression of the $c$ - $L$ fng 
wave of expression, whereas c-hairy1 and c-hairy2 transcription continues in the presence of cyclohexamide (McGrew etal., 1998, Palmeirim et al., 1997). Once initiated, $c$-Lfng mRNA expression moves rapidly anteriorly in the PSM such that the anterior limit of the three genes is swiftly superimposed. A similar lag between her1, her7 and deltaC activation in the PSM has been observed in zebrafish (Gajewski et al., 2003, Oates and Ho, 2002).

Our analysis clearly shows that the duration of the caudal domain of expression differs significantly among the three genes. Both, $c$-Lfng and $c$-hairy 1 mRNAs are degraded fairly rapidly in the caudal PSM. Thus, approximately $20-25 \%$ and $30-35 \%$ of the embryos display a caudal domain of $c$ - $L$ fng and $c$-hairy1 expression, respectively. However, c-hairy2 expression is maintained much longer in this domain, with $65-70 \%$ of the embryos displaying caudal expression (Figure 2D). This variation may be due to differences in mRNA stability among the three genes.

The graphical representation of the expression profile of the three genes clearly shows that the progression of the anterior boundary of these cyclic gene expression domains moves much faster in the caudal PSM than in the rostral PSM. Remarkably, the transition between the two progression modes (fast versus slower) appears to take place in the region of the determination front, which corresponds approximately to the region of somite -IV/V (Dubrulle et al., 2001, Sawada et al., 2001). Caudal to the determination front, PSM cells remain immature in response to FGF8 and they activate their segmentation program only once they become located rostral to the determination front.

At the anterior limit of the PSM, expression of the three genes segregates out into slightly different domains within $S_{0}$, the forming somite and $S_{-1}$ the prospective somite just caudal to $S_{0}$. Thus, as the new border forms, the expression domains of the three genes is such that, $c$-hairy 1 and $c$-hairy 2 are located in the posterior compartment of the newly formed somite, $\mathrm{S}_{1}$, while $c$ $L$ fng is expressed caudal to this new somitic border at the most rostral end of the PSM in the anterior compartment of the next somite to form, $\mathrm{S}_{0}$. It remains to be seen if this late segregation of the three genes to different anteroposterior somitic compartments is related to their respective functions in border formation and/or somite maturation. At least in the case of fringe, studies both in the drosophila wing imaginal disc and in vertebrate limb development have shown that the apposition of fringe positive and fringe negative domains leads to the formation of compartment boundaries (Irvine and Wieschaus, 1994, Panin et al., 1997, Rodriguez-Esteban et al., 1997). A similar model has been proposed for Lfng function during both chick and mouse somite formation (del Barco Barrantes et al., 1999, Sato et al., 2002).

In this report we expand upon previous studies to clarify whether cell-cell contact is required in order to generate synchronized oscillations of cycling gene expression or whether oscillating gene expression is an entirely cell autonomous property of PSM cells. Dissecting the PSM into small fragments, highlighted a number of interesting points in terms of the kinetics of oscillating gene expression in this tissue. Small PSM explants can maintain their synchronized oscillation schedule in the absence of surrounding tissues. However, the intensity of the signal is dramatically reduced when a) the PSM fragments become smaller, b) the time in culture is increased and c) in more posterior fragments of the PSM, to the point that in some cases the caudal domain of expression could not be detected in these small fragments.

The data are consistent with previous findings, which demonstrated that cells of the PSM are not a homogeneous population in terms of their maturation status, but that they are intrinsically endowed with some degree of positional identity with respect to their anteroposterior location along the PSM (Dubrulle et al., 2001, Palmeirim et al., 1998). One factor that plays a key role in this process of maturation specification is FGF8, which has been shown to be instrumental in maintaining a caudal identity in PSM cells (Dubrulle et al., 2001, Sawada et al., 2001). The fact that increased culture time also resulted in diminished expression intensity is consistent with the existence of a permissive factor which is not central to the oscillator mechanism but which is required for maintenance of the oscillations in the PSM. Since culturing the small fragments for an extended time would disrupt and dilute the global FGF gradient this maintenance role could also conceivably be attributed to FGF8.

We observe that once the cells are dissociated and cultured as a cell suspension they no longer exhibit a synchronized expression profile. Thus, a critical cell density is required in order to generate this coordinated, dynamic expression profile. We did nevertheless observe fluctuating percentages of $c$ - $L$ fng positive cells in pools of cells, which were derived from the same PSM explant but which were cultured for different time periods. Such fluctuating percentages are likely to correspond either to a stochastic shut down of oscillating cyclic gene expression or to asynchronous oscillations among cells of the same pool. Given that both the

TABLE 1

\section{ANALYSIS OF CYCLIC GENE EXPRESSION IN ISOLATED PSM CELLS CULTURED IN VITRO}

\begin{tabular}{|c|c|c|c|c|c|c|c|}
\hline \multirow[b]{2}{*}{$c-S h h$} & & 30 ' & $1 \mathrm{~h}$ & 1h30' & $2 \mathrm{~h}$ & 2h30' & $3 \mathrm{~h}$ \\
\hline & 1 & $0 \% ; 272$ & & $0 \% ; 313$ & $0 \% ; 154$ & & \\
\hline \multirow[t]{6}{*}{$c-T b \times 6 L$} & 2 & $95 \% ; 441$ & & $95 \% ; 302$ & $95 \% ; 423$ & & \\
\hline & 3 & $\begin{array}{c}35 \% ; 418 \\
\mathrm{Ph} \mathrm{I}\end{array}$ & & $60 \% ; 280$ & & & \\
\hline & 4 & $\begin{array}{c}10 \% ; 460 \\
\mathrm{Ph} \text { III }\end{array}$ & & $45 \% ; 413$ & & & \\
\hline & 5 & $\begin{array}{c}40 \% ; 105 \\
\text { Ph III-I }\end{array}$ & & $39 \% ; 100$ & & & \\
\hline & 6 & & & & $\begin{array}{c}45 \% ; 176 \\
\text { Ph II }\end{array}$ & & $15 \% ; 161$ \\
\hline & 7 & & & $\begin{array}{c}23 \% ; 103 \\
\text { Ph III }\end{array}$ & & $10 \% ; 238$ & \\
\hline \multirow{5}{*}{$c$-Lfng } & 8 & & & $\begin{array}{c}20 \% ; 138 \\
\text { Ph I }\end{array}$ & $15 \% ; 176$ & & $20 \% ; 307$ \\
\hline & 9 & & & $\begin{array}{c}35 \% ; 188 \\
\text { Ph II }\end{array}$ & $20 \% ; 152$ & $45 \% ; 143$ & \\
\hline & 10 & & $\begin{array}{c}5 \% ; 204 \\
\mathrm{Ph} \mathrm{I}\end{array}$ & & $10 \% ; 555$ & & \\
\hline & 11 & $\begin{array}{c}35 \% ; 351 \\
\text { Ph II }\end{array}$ & & $40 \% ; 603$ & & & \\
\hline & 12 & $\begin{array}{c}\text { 5\%;650 } \\
\text { Ph III }\end{array}$ & & $25 \% ; 584$ & & & \\
\hline
\end{tabular}

Each row corresponds to cell counts from an individual embryo at different time-points (columns). Each panel includes the total cell number counted for that pool and the percentage of cells positive for the gene in question. The panel corresponding to the first time-point for each embryo analyzed for $c$ - $L$ fng expression includes the expression data for the control contralateral explant, which was cultured for the same time. 
Notch receptor and its ligands are transmembrane proteins, activation of the Notch signaling pathway is classically viewed to require cell-cell contact. Notch signaling is therefore likely to be disrupted in the dissociated cells. An arrest of oscillating gene expression would be consistent with the proposed central role played by Notch in the core mechanism of the oscillator.

Alternatively, the existence of desynchronized oscillations in isolated PSM cells would be consistent with a more secondary role for Notch signaling in the co-ordination of the oscillations between cells, as was suggested for the fish embryo (Jiang et al., 2000). Such a possibility implies the existence of a Notchindependent, cell-autonomous mechanism generating the cycling gene oscillations. A recent report implicating a negative feedback loop based on Axin2 and Wnt signaling, acting upstream of the Notch pathway to control of the oscillations, is consistent with this idea (Aulehla et al., 2003). An alternative possibility is that a still unrecognized, cell autonomous pacemaker controls cycling gene oscillations in dissociated PSM cells. The development of real-time imaging techniques to analyze cycling gene expression at single cell resolution will be required to establish whether oscillations are maintained or not in these cell cultures.

\section{Materials and Methods}

\section{Graphical analysis and in situ hybridization}

A large set of stage-matched chick embryos containing 14-18 somites were analyzed by in situ hybridization (Henrique et al., 1995) for expression of $c$-Lfng (Dale et al., 2003), c-hairy1 ( $\mathrm{n}=97)$ or c-hairy2 $(n=87)$. For each of the genes the domains of expression were measured in each embryo, as was the distance of these domains from the last formed somitic boundary. For each gene these values were arranged in order of the advancing limit of the anterior expression limit and then plotted as a graphical representation of the PSM of all the embryos analyzed, using Microsoft Excel. Lfng mRNA expression had been previously analyzed in this manner (Dale et al., 2003). To compare the expression profiles of all three genes the positive domains of expression of the three graphs were superimposed using the window of boundary formation as a cross-reference.

Double in situ hybridization was performed largely as for the single in situ hybridization $(\mathrm{n}=31)$. Modifications included addition of both a digoxigenin and a biotin labeled probe. Incubation with an anti-digoxigenin antibody preceded the first revelation using NBT-BCIP to detect the digoxigenin probe. The embryos were then rinsed before being fixed overnight in $4 \%$ formaldehyde. The embryos were then washed and incubated with an anti-biotin antibody before proceeding with the second revelation using Sigma Fast Red to detect the biotin probe. To avoid biases in the order of color revelation, the expression of each gene was analyzed either with NBT/BCIP or with Sigma Fast Red as the precipitating substrate for detection of alkaline phosphatase activity

\section{In vitro culture of PSM explants}

The caudal region of chick embryos containing 14-18 somites was bisected along the midline. One of these halves was cultured intact on a filter in L15 medium (Gibco) supplemented with 10\% chick serum, 5\% Fetal calf serum, $2.5 \% \mathrm{NaCO}_{3} \mathrm{H}$ and $1 \%$ penicillin/streptomycin solution (Gibco). The other side was micro-dissected along the anteroposterior axis into a number ( 2 to 9 ) of equally sized fragments. These fragments were then cultured for the same time as the intact control half and were then analyzed by in situ hybridization for $c$-Lfng expression.

\section{PSM cell dissociation}

PSM tissue from chick embryos containing 14-18 somites was isolated from one side of the embryo (Palmeirim et al., 1997) and bisected into an anterior and a posterior half along the anteroposterior axis. We focused these analyses only on the posterior PSM because these cells exhibit an all or nothing behavior with respect to cycling gene expression. These cells, $(n=$ approx. 5000-6000), were dissociated by brief exposure to trypsin ( 5 minutes at $37^{\circ} \mathrm{C}$ ) and mechanical pipetting. They were then re-suspended in a large volume $(300 \mu \mathrm{l})$ of a serum-containing medium (see above) to inhibit the trypsin. This cell suspension was split into two to three pools that were each supplemented with $300 \mu \mathrm{l}$ of medium to avoid cell-cell contact and they were then cultured for different lengths of time, varying from 30 to 180 minutes. After fixation (addition of $300 \mu \mathrm{l} 8 \%$ formaldehyde in PBS to the cell suspension for 15 minutes at room temperature), the pools of cells were centrifuged (15 minutes at $3000 \mathrm{rpm}$ ) to plate them onto poly-lysine coated plates. They were subsequently analyzed by in situ hybridization. The number of $c$-Lfng positive cells was recorded as a percentage of the total number of cells counted within each pool. Due to the unavoidable loss of cells at each step of the protocol the number of cells analyzed varied per pool.

\section{Acknowledgements}

We thank J. Dubrulle and A. Reid for comments on the manuscript. This work was supported by a Marie Curie Individual Fellowship from the European Commission to M.M., a Wellcome Prize Travelling Research Fellowship and an EMBO Long term Fellowship to J.K.D. and the laboratory was supported by funding from Centre Nationaldela Recherche Scientifique (CNRS), Human Frontiers Science Programme Organisation (HSFPO), Association francaise contre les myopathies (AFM) and the Université de la Mediterranee-AP de Marseille.

\section{References}

AULEHLA, A. and JOHNSON, R.L. (1999). Dynamic expression of lunatic fringe suggests a link between notch signaling and an autonomous cellular oscillator driving somite segmentation. Dev Biol 207: 49-61.

AULEHLA, A., WEHRLE, C., BRAND-SABERI, B., KEMLER, R., GOSSLER, A., KANZLER, B. and HERRMANN, B.G. (2003). Wnt3a plays a major role in the segmentation clock controlling somitogenesis. Dev Cell 4: 395-406.

BESSHO, Y., HIRATA, H., MASAMIZU, Y. and KAGEYAMA, R. (2003). Periodic repression by the bhlh factor hes7 is an essential mechanism for the somite segmentation clock. Genes Dev 17: 1451-6.

BESSHO, Y. and KAGEYAMA, R. (2003). Oscillations, clocks and segmentation. Curr Opin Genet Dev 13: 379-84.

BESSHO, Y., SAKATA, R., KOMATSU, S., SHIOTA, K., YAMADA, S. and KAGEYAMA R. (2001). Dynamic expression and essential functions of hes7 in somite segmentation. Genes Dev 15: 2642-7.

CONLON, R.A., REAUME, A.G. and ROSSANT, J. (1995). Notch1 is required for the coordinate segmentation of somites. Development. 121: 1533-1545.

DALE, J.K., MAROTO, M., DEQUEANT, M.L., MALAPERT, P., MCGREW, M. and POURQUIÉ, O. (2003). Periodic notch inhibition by lunatic fringe underlies the chick segmentation clock. Nature 421: 275-8.

DALE, K.J. and POURQUIÉ, O. (2000). A clock-work somite. Bioessays 22: 72-83.

DEL BARCO BARRANTES, I., ELIA, A., WUNNSCH, K., HRABDE DE ANGELIS, M. MAK, T., ROSSANT, J., CONLON, R., GOSSLER, A. and LUIS DE LA POMPA, J. (1999). Interaction between notch signalling and lunatic fringe during somite boundary formation in the mouse. Curr. Biol. 9: 470-480.

DUBRULLE, J., MCGREW, M.J. and POURQUIÉ, O. (2001). Fgf signaling controls somite boundary position and regulates segmentation clock control of spatiotemporal hox gene activation. Cell 106: 219-32.

EVRARD, Y.A., LUN, Y., AULEHLA, A., GAN, L. and JOHNSON, R.L. (1998). Lunatic fringe is an essential mediator of somite segmentation and patterning Nature 394: 377-381.

FORSBERG, H., CROZET, F. and BROWN, N.A. (1998). Waves of mouse lunatic fringe expression, in four-hour cycles at two-hour intervals, precede somite boundary formation. Curr.Biol. 8: 1027-1030.

FREITAS, C., RODRIGUES, S., CHARRIER, J.B., TEILLET, M.A. and PALMEIRIM, I. (2001). Evidence for medial/lateral specification and positional information within the presomitic mesoderm. Development 128: 5139-47. 
GAJEWSKI, M., SIEGER, D., ALT, B., LEVE, C., HANS, S., WOLFF, C., ROHR, K.B. and TAUTZ, D. (2003). Anterior and posterior waves of cyclic her1 gene expression are differentially regulated in the presomitic mesoderm of zebrafish. Development 130: 4269-4278.

HENRIQUE, D., ADAM, J., MYAT, A., CHITNIS, A., LEWIS, J. and ISH-HOROWICZ, D. (1995). Expression of a delta homologue in prospective neurons in the chick. Nature 375: 787-790.

HIRATA, H., YOSHIURA, S., OHTSUKA, T., BESSHO, Y., HARADA, T., YOSHIKAWA, K. and KAGEYAMA, R. (2002). Oscillatory expression of the bhlh factor hes 1 regulated by a negative feedback loop. Science 298: 840-843.

HOLLEY, S.A., GEISLER, R. and NUSSLEIN-VOLHARD, C. (2000). Control of her1 expression during zebrafish somitogenesis by a delta-dependent oscillator and an independent wave-front activity. Genes Dev 14: 1678-90.

HOLLEY, S.A., JULICH, D., RAUCH, G.J., GEISLER, R. and NUSSLEIN-VOLHARD, C. (2002). Her1 and the notch pathway function within the oscillator mechanism that regulates zebrafish somitogenesis. Development 129: 1175-83.

HRABE DE ANGELIS, M., MCINTYRE, J. and GOSSLER, A. (1997). Maintenance of somite borders in mice requires the delta homologue dii1. Nature 386: 717721.

IRVINE, K.D. and WIESCHAUS, E. (1994). Fringe, a boundary-specific signaling molecule, mediates interactions between dorsal and ventral cells during drosophila wing development. Cell 79: 595-606

JIANG, Y.J., AERNE, B.L., SMITHERS, L., HADDON, C., ISH-HOROWICZ, D. and LEWIS, J. (2000). Notch signalling and the synchronization of the somite segmentation clock. Nature 408: 475-9.

JOUVE, C., PALMEIRIM, I., HENRIQUE, D., BECKERS, J., GOSSLER, A., ISHHOROWICZ, D. and POURQUIÉ, O. (2000). Notch signalling is required for cyclic expression of the hairy-like gene hes 1 in the presomitic mesoderm. Development 127: 1421-1429.

KUSUMI, K., SUN, E.S., KERREBROCK, A.W., BRONSON, R.T., CHI, D.C., BULOTSKY, M.S., SPENCER, J.B., BIRREN, B.W., FRANKEL, W.N. and LANDER, E.S. (1998). The mouse pudgy mutation disrupts delta homologue dII3 and initiation of early somite boundaries. Nat Genet 19: 274-8.

LEIMEISTER, C., DALE, K., FISCHER, A., KLAMT, B., HRABE DE ANGELIS, M., RADTKE, F., MCGREW, M.J., POURQUIÉ, O. and GESSLER, M. (2000). Oscillating expression of c-hey2 in the presomitic mesoderm suggests that the segmentation clock may use combinatorial signaling through multiple interacting bhlh factors. Dev Biol 227: 91-103.

MAROTO, M. and POURQUIÉ, O. (2001). A molecular clock involved in somite segmentation. Curr Top Dev Biol 51: 221-48.
MCGREW, M.J., DALE, J.K., FRABOULET, S. and POURQUIÉ, O. (1998). The lunatic fringe gene is a target of the molecular clock linked to somite segmentation in avian embryos. Curr Biol 8: 979-82.

OATES, A.C. and HO, R.K. (2002). Hairy/e(spl)-related (her) genes are central components of the segmentation oscillator and display redundancy with the delta/notch signaling pathway in the formation of anterior segmental boundaries in the zebrafish. Development 129: 2929-46.

OKA, C., NAKANO, T., WAKEHAM, A., DE LA POMPA, J.L., MORI, C., SAKAI, T., OKAZAKI, S., KAWAICHI, M., SHIOTA, K., MAK, T.W. et al. (1995). Disruption of the mouse rbp-j kappa gene results in early embryonic death. Development. 121: 3291-3301.

PALMEIRIM, I., DUBRULlE, J., HENRIQUE, D., ISH-HOROWICZ, D. and POURQUIÉ, O. (1998). Uncoupling segmentation and somitogenesis in the chick presomitic mesoderm. Dev Genet. 23: 77-85.

PALMEIRIM, I., HENRIQUE, D., ISH-HOROWICZ, D. and POURQUIÉ, O. (1997). Avian hairy gene expression identifies a molecular clock linked to vertebrate segmentation and somitogenesiss. Cell 91: 639-648.

PANIN, V.M., PAPAYANNOPOULOS, V., WILSON, R. and IRVINE, K.D. (1997). Fringe modulates notch-ligand interactions. Nature 387: 908-12.

POURQUIÉ, O. (2003). The segmentation clock: Converting embryonic time into spatial pattern. Science. 301: 328-30.

POURQUIÉ, O. and TAM, P.P. (2001). A nomenclature for prospective somites and phases of cyclic gene expression in the presomitic mesoderm. Dev Cell 1:61920.

RODRIGUEZ-ESTEBAN, C., SCHWABE, J.W., DE LA PENA, J., FOYS, B., ESHELMAN, B. and BELMONTE, J.C. (1997). Radical fringe positions the apical ectodermal ridge at the dorsoventral boundary of the vertebrate limb [published erratum appears in nature 1997 aug 28;388(6645):906]. Nature 386: 360-366.

SATO, Y., YASUDA, K. and TAKAHASHI, Y. (2002). Morphogical boundary forms by a novel inductive enevt mediated by lunatic fringe and notch during somitic segmentation. Development 129: 3633 - 3644.

SAWADA, A., SHINYA, M., JIANG, Y.J., KAWAKAMI, A., KUROIWA, A. and TAKEDA, H. (2001). Fgf/mapk signalling is a crucial positional cue in somite boundary formation. Development 128: 4873-80.

SWIATEK, P.J., LINDSELL, C.E., DEL AMO, F.F., WEINMASTER, G. and GRIDLEY, T. (1994). Notch1 is essential for postimplantation development in mice. Genes Dev 8: 707-719.

ZHANG, N. and GRIDLEY, T. (1998). Defects in somite formation in lunatic fringedeficient mice Nature 394: 374-377. 